\title{
Analysis and Synthesis of Coupled Microstrip Circuits with More Than Two Coupled Strips
}

Dalby, Arne Brejning

Published in:

7th European Microwave Conference

Link to article, DOI:

10.1109/EUMA.1977.332464

Publication date:

1977

Document Version

Publisher's PDF, also known as Version of record

Link back to DTU Orbit

Citation (APA):

Dalby, A. B. (1977). Analysis and Synthesis of Coupled Microstrip Circuits with More Than Two Coupled Strips. In 7th European Microwave Conference (pp. 441-445). IEEE. https://doi.org/10.1109/EUMA.1977.332464

\section{General rights}

Copyright and moral rights for the publications made accessible in the public portal are retained by the authors and/or other copyright owners and it is a condition of accessing publications that users recognise and abide by the legal requirements associated with these rights.

- Users may download and print one copy of any publication from the public portal for the purpose of private study or research.

- You may not further distribute the material or use it for any profit-making activity or commercial gain

- You may freely distribute the URL identifying the publication in the public portal 
Arne B. Dalby

ABSTRACT. It is shown that the $\mathrm{Y}$-parameters for the $2 \mathrm{~N}$-port network, $\mathrm{N}$ coupled strips form, may be found using almost arbitrary quasi-propagation modes if the relative bandwith of interest is small. Chosing a special set of propagation modes leads to simple expressions for the $y$-parameters, which are easy to use in an iterative synthesis procedure. The use of quasi-modes also facilitates the calculation of primary parameters (capacitances and phasevelocities). A further advantage is that empirical formulas may be used. Such formulas were developed, which give surprisingly accurate results.

INTRODUCTION. The expressions for network parameters, as found in e.g. [1-3] for a coupled microstrip network, will normally be so complex that they are difficult if not impossible to use in a synthesis procedure. Another problem is the calculation of primary parameters. This is a very timeconsuming process if accurate results are wanted. The existing fast methods e.g. [4] based on empirical formulas are too inaccureate, and can only be used on two identical coupled strips. Solutions to both problems are given in this paper, resulting in a fast microstrip analysis program and a synthesis program for interdigital bandpass filters.

Y-PARAMETERS. N coupled strips form a $2 \mathrm{~N}$-port network, and the $\mathrm{y}$-parameters for this network may be found knowing the capacitance and inductance matrices for the strips. It is assumed that only the $\mathrm{N}$ fundamental propagation modes exist on the system, and that each of these is a lossfree TEM mode. The relationship between voltages and currents in a transverse plane $(x, y)$ (fig.1) may be written

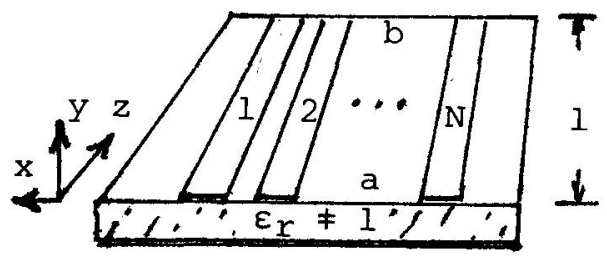

$\mathrm{N}$ COUPLED MICROSTRIPS. fig. 1

$$
\gamma U=j \omega L I \text { and } \gamma I=j \omega C U
$$

$U$ and I are column vectors representing the voltages and currents on the strips. L and $C$ are the $\mathrm{NxN}$ square inductance and capacitance matrices. $\gamma$ $=j \beta=j \omega / v$ is a mode propagation constant, where $v$ is the phasevelocity. one solution to (I) is

$$
I=C T_{u} \vee T_{u}^{-1} U
$$

$T_{u}=\left\{T_{u l}, T_{u 2}, \cdots T_{u N}\right\}$ is a NxN matrix, where $T_{u j}$ is an eigenvector associated with the eigenvalue $1 / v_{j}^{2}$ for the matrix $L C,[1-3]$. V is a diagonal matrix with diagonal elements $=v_{j}$. In (2) propagation in the z-direction only is assumed. From (2) and the results known from transmissionline theory, the relationship between voltages on and currents into the $2 \mathrm{~N}$-port network, the $\mathrm{N}$ strips form, can be found as

where

$$
\begin{aligned}
& \left\{\begin{array}{l}
I_{a} \\
I_{b}
\end{array}\right\}=\left\{\begin{array}{ll}
Y_{a a} & Y_{a b} \\
Y_{b a} & Y_{b b}
\end{array}\right\}\left\{\begin{array}{l}
U_{a} \\
U_{b}
\end{array}\right\} \\
& Y_{a a}=Y_{b b}=C T_{u}\{-j v \cot (\omega l / v)\} T_{u}^{-1}
\end{aligned}
$$

Engineering Academi of Denmark, Department of Electrical Engineering Building 451, 2800 Lyngby, Denmark. 


$$
Y_{\mathrm{ab}}=Y_{\mathrm{ba}}=C T_{\mathrm{u}}\{j \mathrm{v} \csc (\omega \mathrm{l} / \mathrm{v})\} \mathrm{T}_{\mathrm{u}}^{-1}
$$

$\{-j \vee \cot (\omega l / v)\}$ is a diagonal matrix where the diagonal elements are $=$ $-j v_{j} \cot \left(\omega l / v_{j}\right)$. Similarly for $\{j v \csc (\omega l / v)\}$. The indeces $a$ and $b$ refer to the two ends of the circuit. A more convenient form of $(4,5)$ for the purpose of introducing quasi modes is

$$
\begin{aligned}
& Y_{a a}=Y_{b b}=\left\{-j Y_{i j} T_{u i j} \cot \left(\omega l / v_{j}\right)\right\} T_{u}^{-1} \\
& Y_{a b}=Y_{b a}=\left\{j Y_{i j} T_{u i j} \csc \left(\omega l / v_{j}\right)\right\} T_{u}^{-1}
\end{aligned}
$$

$\left\{-j \mathrm{Y}_{i j} \mathrm{~T}_{\mathrm{uij}} \cot \left(\omega \mathrm{l} / \mathrm{v}_{j}\right)\right\}$ and $\left\{j \mathrm{Y}_{i j} \mathrm{~T}_{\mathrm{uij}} \csc \left(\omega l / \mathrm{v}_{j}\right)\right\}$ are NxN matrices where

$$
\mathrm{Y}_{i j}=\left(\mathrm{v}_{j} \sum_{\mathrm{r}=1}^{\mathrm{N}} \mathrm{C}_{i r \mathrm{Trj}}\right) / \mathrm{T}_{u i j}=c_{i j} \mathrm{v}_{i j}
$$

$\mathrm{Y}_{i j}$ is the characteristic admittance for strip $i$ in mode $j . c_{i j}$ is the apparent stripcapacitance in this mode.

QUASI MODES. Letting $U_{a}=k Q_{J}$ and $U_{b}=0$, where $Q_{J}$ is an arbitrary column vector representing a quasi mode $\mathrm{J}$. give for e.g. Ia

$$
I_{a}=\left\{-j Y_{i j}^{T}{ }_{u i j} \cot \left(\omega l / v_{j}\right)\right\} T_{u}^{-1} k Q_{J}
$$
$T_{u}^{-1} Q_{J}$ is a column vector, which for clarity will be called $Q$. The current
on e.g. strip n at $z=0$ is

$$
I_{n J a}=k\left(-j \sum_{r=1}^{N} Y_{n r} T_{u n r} \cot \left(\omega l / v_{r}\right) q_{r}\right)
$$

For $\omega l / v_{r} \simeq \pi / 2, \cot \left(\omega l / v_{r}\right) \simeq \pi / 2-\omega l / v_{r}$. This gives

$$
\begin{aligned}
I_{n J a} & \simeq k\left(-j \pi / 2 \sum_{r=1}^{N} Y_{n r} T_{u n r} q_{r}+j \omega l \sum_{r=1}^{N} C_{n r} T_{u n r} q_{r}\right) \\
& =k Q_{n J}\left(-j \pi / 2 Y_{n J}^{\prime}+j \omega I c_{n J}^{\prime}\right)
\end{aligned}
$$

where

$$
\begin{aligned}
& \mathrm{Y}_{\mathrm{nJ}}^{\prime}=\left(\sum_{\mathrm{r}=1}^{N} \mathrm{Y}_{n r^{T}} \mathrm{unr}_{\mathrm{r}} \mathrm{q}_{\mathrm{r}}\right) / \mathrm{Q}_{\mathrm{nJ}}=\left(\sum_{r=1}^{\mathrm{N}} \mathrm{C}_{\mathrm{n}_{r}} \mathrm{v}_{\mathrm{r}} \mathrm{T}_{\mathrm{unr}} \mathrm{q}_{\mathrm{r}}\right) / \mathrm{Q}_{\mathrm{nJ}} \\
& c_{n J}^{\prime}=\left(\sum_{r=1}^{N} c_{n r} T^{T} u_{r}{ }^{q_{r}}\right) / Q_{n J}=\left(\sum_{s=1}^{N} c_{n s} Q_{s J}\right) / Q_{n J}
\end{aligned}
$$

Introducing $\cot \left(\omega l / \mathrm{v}_{n J}\right)$ in (12), where $\mathrm{v}_{\mathrm{nJ}}=\mathrm{Y}_{\mathrm{nJ}}^{\prime} / \mathrm{C}_{\mathrm{nJ}}^{\prime}$ gives

$$
I_{n J a} \simeq \mathrm{k}_{\mathrm{nJ}}\left(-j \mathrm{Y}_{\mathrm{nJ}}^{\prime} \cot \left(\omega \mathrm{l} / \mathrm{v}_{\mathrm{nJ}}\right)\right)
$$

which is the expression for $I_{n J a}$ if $Q_{J}$ had represented a true mode. Using (15) instead of (10) will not give rise to any serious errors $(<2 \%)$ if $v_{r}$, $r=1,2 \cdots \mathrm{N}$, and $\mathrm{v}_{\mathrm{nJ}}$ all lie inside an interval of $20 \%$ relative magnitude, and $0<\omega l / v_{n J}<1.2 \pi / 2$. The admittance $Y_{n J}^{\prime}$ and the capacitance $C_{n J}^{\prime}$ are the characteristic admittance and the apparent strip capacitance for strip $\mathrm{n}$ in mode $J$. Observe that $c_{n J}^{\prime}$ is a sum of capacitances, and that $Y_{n J}^{\prime}$ is the sum (parallel connection) of the corresponding admittances. This fact is utilized in the empirical formulas used to find the primary parameters (see later). If $\mathrm{N}$ linearly independant but otherwise arbitrary column vectors $Q_{J}$ are chosen, $\mathrm{J}=1,2 \cdots \mathrm{N}$, the $\mathrm{y}$-parameters may be approximated by

$$
\begin{aligned}
& Y_{a a}=Y_{b b}=\left\{-j Y_{I J}^{\prime} Q_{I J} \cot \left(\omega l / v_{I J}\right)\right\} Q^{-1} \\
& Y_{\mathrm{ab}}=Y_{\mathrm{ba}}=\left\{j \mathrm{Y}_{\mathrm{IJ}}^{\prime} \mathrm{Q}_{I J} \csc \left(\omega 1 / \mathrm{v}_{I J}\right)\right\} Q^{-1}
\end{aligned}
$$

$Q$ is a NxN matrix representing the $N$ quasi modes, $Q=\left\{Q_{1}, Q_{2} \cdot Q_{N}\right\} \cdot(16,17)$ are almost identical to $(6,7)$, but an important difference is that NxN phasevelocities must be known when using $(16,17)$, instead of only N. This may seem a serious complication, but it turns out not to be so. Another difference is that $Y_{a a}$ etc. in $(16,17)$ are not symmetrical, which they should be 
because of reciprocity. However, the errors introduced by using $(16,17)$ are quite small inside the frequency band of interest, and can be reduced even further by taking $Y_{a a}$ as the mean between $Y_{a a}$ and $Y_{a a}^{T}$ etc. This also ensures reciprocity.

ANALYSIS AND SYNTHESIS. The quasi modes should be chosen so that a synthesis procedure and thereby also analysis is made easy. Such a choise is

$$
Q=\left\{\begin{array}{rrrrr}
1 & -1 & -1 & \ldots & -1 \\
1 & 1 & -1 & & -1 \\
\cdot & & & & \cdot \\
1 & 1 & 1 & & 1
\end{array}\right\}, \quad Q^{-1}=\left\{\begin{array}{rrrrr}
\frac{1}{2} & 0 & 0 & \ldots & \frac{1}{2} \\
-\frac{1}{2} & \frac{1}{2} & 0 & & 0 \\
0 & -\frac{1}{2} & \frac{1}{2} & & 0 \\
0 & 0 & 0 & -\frac{1}{2} & \frac{1}{2}
\end{array}\right\}
$$

From (16-18) it can be seen that each y-parameter will consist of only two terms instead of $\mathrm{N}$. Ignoring coupling between nonadjacent strips give further simplifications. Only three different modes need be considered for each strip, thus reducing the number of different $\mathrm{Y}_{\text {IJ }}^{\prime}$ and $\mathrm{v}_{\mathrm{IJ}}$ to $3 \mathrm{xN}$. In fig.2 the three modes and their parameters are shown.

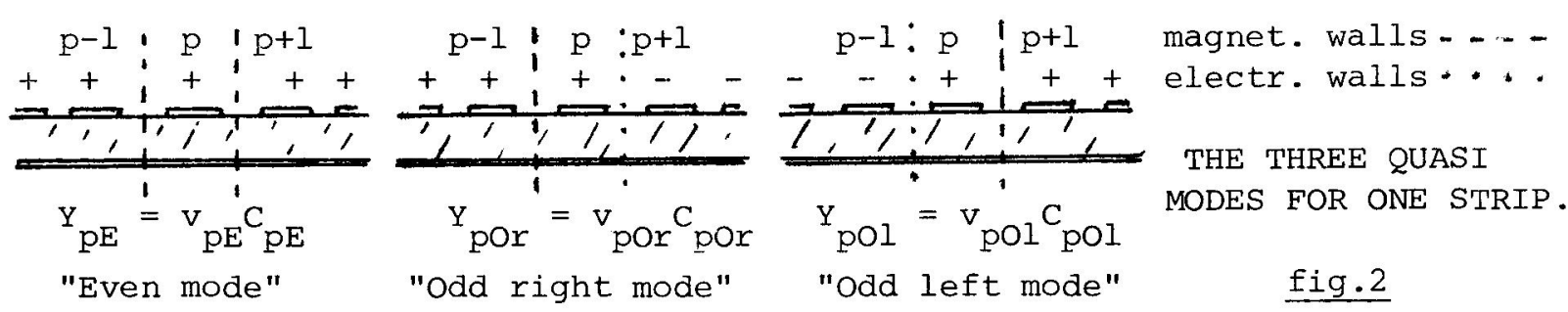

From fig.2 it can be seen that only two different procedures are needed to calculate the primary parameters, because the two odd-modes can be evaluated using the same procedure. A further advantage is that the $y$-parameters may be calculated for one strip at a time, virtually independant of the other strips.

A 5TH ORDER INTERDIGITAL BANDPASS FILTER. To illustrate the use of quasimodes, the synthesis procedure for an interdigital filter will be briefly outlined. In fig.3 a 7-strip 5th order bandpass filter is shown schematically along with an equivalent circuit based on the $y$-parameters. The filter is only a 5 th order filter because yllaa and Y77aa $\ll 1 / z_{0}$.
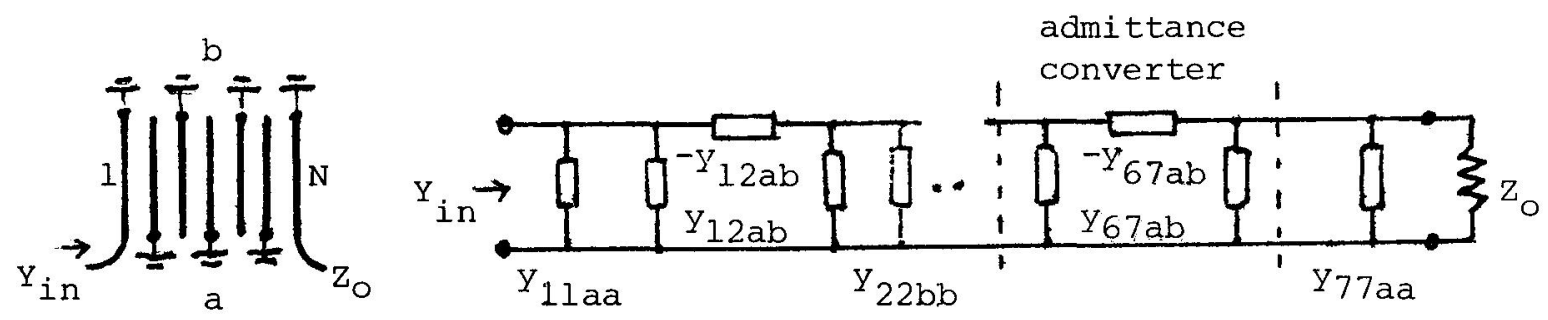

fig. 3 th ORDER INTERDIGITAL FILTER AND ITS EQUIVALENT CIRCUIT.

If the striplengths are adjusted so that $\mathrm{y}_{11 \mathrm{aa}}, \mathrm{y}_{22 \mathrm{bb}} \cdots \mathrm{y}_{77 \mathrm{aa}}$ are all 0 at the centre frequency $f_{0}$ for the filter, we have from (16-18) and fig.2

$$
\begin{aligned}
& y_{\text {ppaa }}=-j \frac{1}{2}\left(v_{p O 1} C_{p O l}+v_{p o r} C_{p o r}\right) \frac{\pi B W}{2 \omega_{O}}=k_{p} s^{\prime} \\
& y_{p, p-l a b}=j \frac{1}{2}\left(v_{p E} C_{p E}-v_{p O l} C_{p O l}\right)=J_{p, p-1} \\
& \text { etc. at frequencies near } f_{o} \cdot \\
& \mathrm{s}^{\prime}=j\left(\omega-\omega_{0}\right) / B W, B W=\text { bandwidth in } \mathrm{rad} / \mathrm{sec}
\end{aligned}
$$


The synthesis of the $\mathrm{K}_{\mathrm{p}}$ 's and the $\mathrm{J}_{\mathrm{p}, \mathrm{p}-\mathrm{l}} \mathrm{s}$ to obtain the desired filter response is classical and described in e.g. [5]. But to find the stripwidths and spacings, an iterativ procedure must be used. This turns out to be quite simple, because the J's depend mainly on the corresponing spacings and the K's mainly on the stripwidths. An iterative procedure for symmetric interdigital filters of the kind shown in fig. 3 was developed, which in a few runs give a possible combination of strip-widths and -spacings. Because there are infinitely many solutions, some of the dimensions must be chosen beforehand to ensure a practical solution.

A 5 th. order filter was synthesized as a Chebyshev filter with 1 dB ripple, $f_{O}=670 \mathrm{MHz}$ and relative bandwidth $=0.2$. To account for nonideal shortcircuits and end-effects, the actual strip lengths were made $3.7 \mathrm{~mm}$ shorter than the calculated lengths. The filter and its response is shown in fig.4. The calculated response, which resembles the measured response quite remarkably, was calculated by a computer program using a standard network analysis procedure on the equivalent circuit in fig. 3. The analysis program can be used on almost any kind of linear microstrip circuit, because lumped elements, shortcircuits and equivalent circuits for e.g. transistors may be introduced into the equivalent circuit for the microstrips.

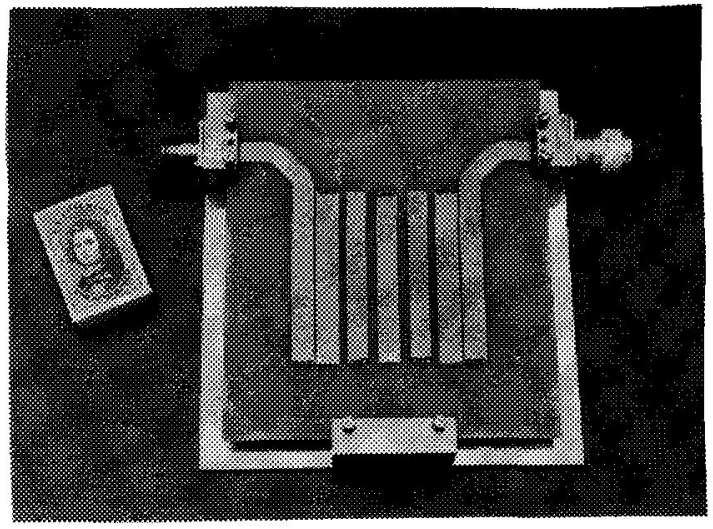

fig. 4 th ORDER INTERDIGITAL BANDPASS FILTER AND ITS RESPONSE :

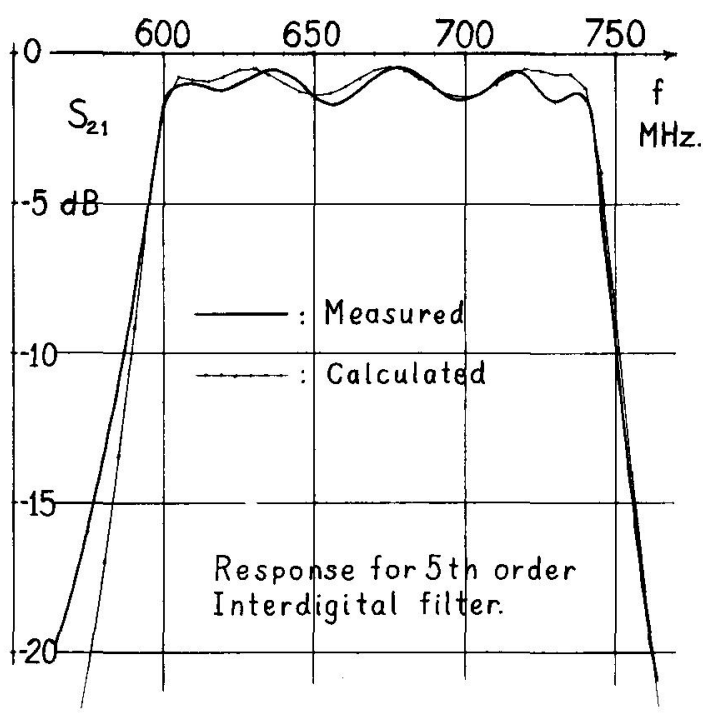

EMPIRICAL FORMULAS. In the analysis- and synthesis programs mentioned above empirical formulas are used to calculate the parameters $Y_{p E}, v_{p E}$ etc. These formulas can be used for practical values of physical dimensions and dielectric constant $\left(I<\varepsilon_{r}<16\right)$. It is beyond the scope of this paper to describe the development of these formulas, but the capacitances $C_{p E}$, $C_{p 0 l}$ etc. are found as parallel connections of capacitors, each corresponding to some part of the electrical field around the strips, and the admittances $\mathrm{Y}_{\mathrm{pE}}$, $\mathrm{Y}_{\mathrm{p} 01}$ etc. are found as parallel connections of admittances corresponding to the same capacitors. Doing it this way, it is possible to obtain results, which vary in accordance with physical reasoning, when some dimension is changed. This is very important, and very difficult to obtain using other methods, unless unduly much computertime is used.

In fig. 5 and 6 even- and odd mode parameters are given for two identical coupled strips and $\varepsilon_{r}=10$. Comparing these results with the results obtained by e.g. Bryant and weiss [6] show good agreement. There are, however, differences between the phasevelocities (a few 8 ). The curves in fig.6, giving even- and odd mode velocities for different combinations of stripwidths and -spacings, show that the empirical formulas give phasevelociti- 
es that vary in a way predictable by physical reasoning. As a control on the formulas, the even- and odd mode inductances for two identical coupled strips were found for $1<\varepsilon_{r}<15$. They vary \pm 1.28 in this interval. They should be indepenant of $\varepsilon_{r}$. These results were obtained for relative stripwidths and -spacings lying in the interval 0.2-5.

CONCLUSION. A set of "tools" have been developed which can be used to analyse and synthesize coupled microstrip circuits with more than two strips.

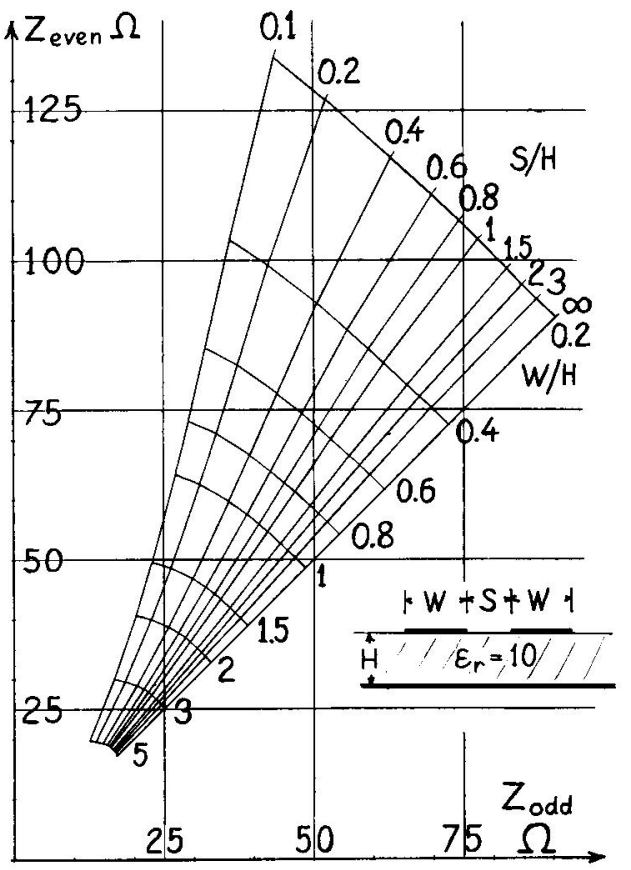

$\underline{\text { fig. }} 5$

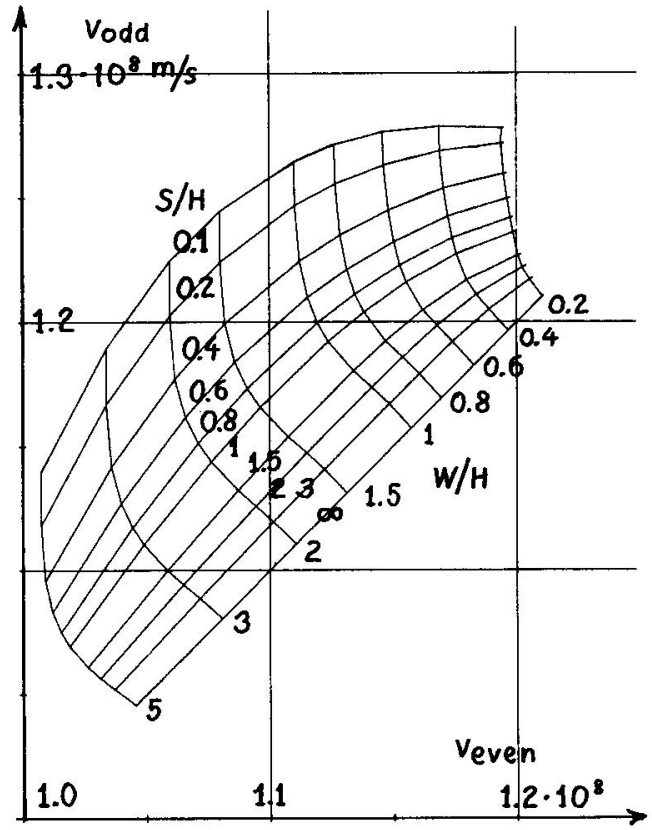

$\underline{\mathrm{fig} .6}$

\section{REFERENCES.}

1. Roland Briechle: Obertragungseigenschaften gekoppelter, verlustbehafteter Mehrleitersysteme mit geschichtetem Dielektrikum. FREQUENZ 29/3 1975

2. Roland Briechle: Analyse von Kammleitungsfiltern und ähnlichen Baugruppen aus gekoppelten Mehrleitersystemen. FREQUENZ 29/4 1975.

3. Kenneth D. Marx: Propagation Modes, Equivalent Circuits, and Characteristic Terminations for Multiconductor Transmission Lines with inhomogeneous Dielectrics. IEEE Transact. MTT 21/7 1973.

4. Sina Akhtarzad et.al.: The design of coupled Microstrip Lines. IEEE Transact. MTT 23/6 1975.

5. R.E. Collin: Foundations for Microwave Engineering. McGraw-Hill 1966.

6. Bryant and Weiss: Parameters of Microstrip Transmission Lines and of coupled Pairs of Microstrip Lines. IEEE Transact. MTT 16/12 1968. 